\title{
Shape-Assisted Self-Assembly
}

Authors: Joseph F. Woods ${ }^{\dagger, 1}$, Lucía Gallego ${ }^{\dagger, 1}$, Pauline Pfister ${ }^{1}$, Mounir Maaloum ${ }^{2}$, Andreas Vargas Jentzsch ${ }^{\dagger}, 2$, Michel Rickhaus ${ }^{1 *}$

\section{Affiliations:}

${ }^{1}$ Department of Chemistry, University of Zurich; Zurich, Switzerland.

${ }^{2}$ SAMS Research Group, Université de Strasbourg, CNRS, Institute Charles Sadron; Strasbourg, France.

$\dagger^{\dagger}$ these authors contributed equally

*Corresponding author. Email: michel.rickhaus@chem.uzh.ch

\begin{abstract}
Self-assembly and molecular recognition are critical processes both in life and material sciences. They usually depend on strong, directional non-covalent interactions to gain specificity and to make long-range organization possible. Most supramolecular constructs are also at least partially governed by topography, whose role is hard to disentangle. This makes it nearly impossible to discern the potential of shape and motion in the creation of complexity. Here, we demonstrate that long-range order in supramolecular constructs can be driven by the topography of the individual units even in the absence of directional interactions. Here, molecular units of remarkable simplicity self-assemble in solution to give homogeneous singlemolecule thin two-dimensional supramolecular polymers of defined boundaries. This dramatic example spotlights the critical function that topography can have in molecular assembly and paves the path to rationally designed systems of increasing sophistication.
\end{abstract}

One-Sentence Summary: Topography as a molecular design principle permits the construction of otherwise inaccessible supramolecular structures. 


\section{Main Text:}

Shape plays a critical role in natural molecular recognition processes such as enzyme catalysis. As early as 1894, Emil Fischer proposed with his 'Lock and Key' model that a substrate must possess a profile complementary to that of the enzymatic cleft; otherwise, there is poor association between the two (1). Within this understanding, one of the simplest topographical elements that can allow for (molecular) recognition is curvature. The notion of curvature has an underlying and accepted role in creating order within supramolecular systems but is overshadowed by the driving forces for (self-)assembly $(2,3)$. Despite its relevance, the role of topography, in the absence of any other contributing factors, as an element of recognition in supramolecular assembly processes has long eluded experimental proof, and little is known about the unique advantages exploiting it would bring forward.

Over three decades ago, the first reports on supramolecular polymers $(4,5)$ catapulted the interest in soft dynamic materials and, particularly, hydrogen-bonded linear assemblies (6-9). Together with other highly directional non-covalent interactions (10-13) they remain the classical choice for designing self-assembling systems due to their reversible and self-healing properties $(14,15)$. Less directional non-covalent interactions between $\pi$-surfaces or hydrophobic contacts seldom result in assemblies (16-18). In the rare cases when they do, assistance is required from another effect in tandem (19-21) because of the limited long-range control. To a certain extent, curvature has been recognized as a feature for assembly-proficient monomers. For instance, Aida and co-workers exploited a bowl-shaped corannulene for permitting the creation of dormant supramolecular species and thus the development of living-supramolecular polymerization (22). Moreover, they recently have used saddle-shaped molecules to report the first example of alternating heterochiral supramolecular copolymerization (23). Additionally, Itami demonstrated that curved nanographenes can associate to form nanofibers purely based on dipolar $\pi-\pi$ stacking (24). These rare examples offer a small glimpse of the scope that this approach towards developing functional materials has to offer.

Herein, we report that in the absence of directional non-covalent interactions, the shape of a molecular unit can become the governing ordering force to assemble micrometer-long stacks. Those columns then self-assemble into two-dimensional (2D) sheets of single-molecular thickness and highly defined boundaries. These nanostructures are formed by assembly in solution of a negatively curved molecule and held together primarily by dipolar $\pi-\pi$ interactions. The simplicity of this approach towards 2D materials is unique and contrasts strongly with existing strategies for which a representative overview is presented in Fig. S1. Notably, this approach is complementary to on-surface self-assembly (25) and surface nucleated thin films (26) because the nanostructures exist and are persistent in solution. The uniqueness of this process, which we term 'shape-assisted self-assembly', demonstrates the significance of shape in the self-assembly process, provides a crucial conceptual tool and brings us closer to control and rational design of complex bottom-up assemblies in nanotechnology in general.

Flat molecules are frequently observed in supramolecular polymerizations due to ease of access to these structures synthetically $(8,27,28)$. Bowl shapes $(29,30)$, and more so saddles $(24,31$, 32 ), are far less commonly used topographies for the opposite reason. Yet, to introduce order in an assembly, negatively curved systems stand out as preferred candidates to induce eclipsed stacking because transverse rigidity is increased on both molecular and macroscopic scales (23, $24,33)$. Asymmetry in saddle design, in addition to replacing a uniform surface with a framework scaffold, heightens the entropic barriers further due to unequal axes of principal curvature (Fig. 1). An energetic minimum can then be reached with eclipsing negatively curved 
frames such that small deviations from overlap demand a high energetic penalty and, consequently, enforce order. Depending on the asymmetry of the saddle, their assemblies likely present intrinsically differentiated lateral interactions permitting the formation of tertiary structures. Müllen and co-workers (34) first reported a small saddle-shaped molecule that possesses these characteristics within the aryl frame. Remanufacturing of this core with different functional groups could serve as a monomer suitable for such polymerization (Fig. 1).

As a first approach, peripheral alkyl substitution of four of the six available sites was proposed to (i) enhance solubility, and (ii) favor eclipsing $\pi$-surfaces. The addition of hydrogen bonding motifs was deliberately avoided because of their superior capability to order systems (23). As reference systems, we included the unfunctionalized porphyrinoid to investigate the effect of a core without sidechains and the macrocycle bearing tert-butyl groups due to its reduced propensity to self-assemble (34).

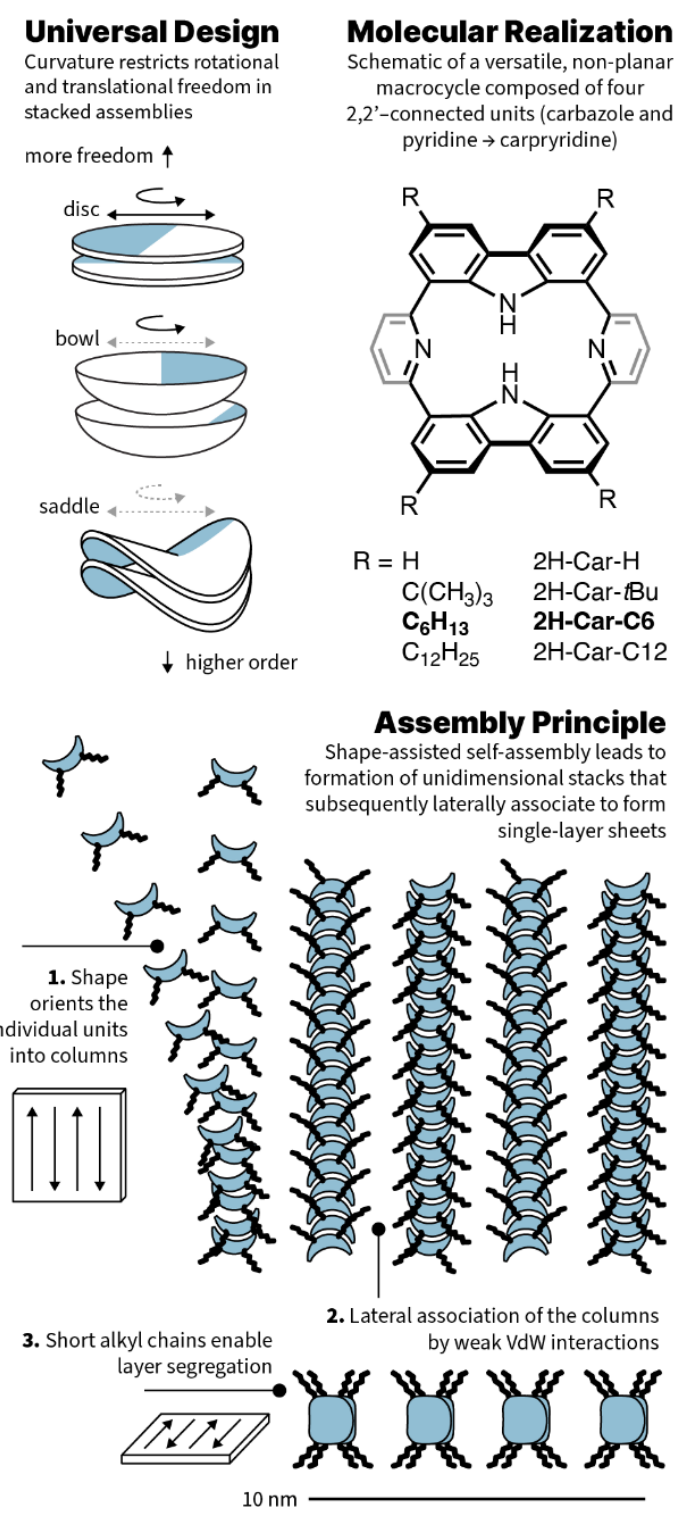


Fig. 1 Design principle, structural scheme and assembly process. (left) Shape as a design principle to restrict rotational and translational freedom. (right) Generic chemical structure of carpyridines with $\mathrm{R}$ group modifications to the core. (bottom) Multi-stage assembly process, where (i) units assemble into linear stacks guided by the shape of their core and driven by entropy before (ii) assembling into defined nanosheets.

Based on Miyaura borylation of dibromocarbazoles prior to macrocyclization, we developed a unifying synthetic protocol. Four-fold Suzuki-Miyaura cross-coupling with a commercial pyridine gave cyclic products (up to $13 \%$ yield; $60 \%$ yield per coupling) that we name 'carpyridine' as a consequence of their interlinked constituents. Although all carpyridines could be synthesized using this route, preparation of the unsubstituted derivative $\mathbf{2 H}$-Car-H resulted in inferior yields $(<1 \%)$. By subjecting $\mathbf{2 H}$-Car- $\boldsymbol{t}$ Bu to a reverse Friedel-Crafts alkylation reaction, we could efficiently remove the tert-butyl groups from the rim of the macrocycle ( $75 \%$ yield). Full characterization of the synthesized compounds and their intermediates is described in the SI.

All compounds showed excellent solubility and stability in common organic solvents such as chlorinated solvents or THF. In apolar solvents like toluene or methylcyclohexane (MCH) the solubility was visibly reduced. This was corroborated by variable temperature ${ }^{1} \mathrm{H}$ NMR which displayed an entirely different behavior as a function of the solvent used. 2H-Car-C6 (7.5 mM) in deuterated 1,1,2,2-tetrachloroethane (TCE- $\left.d_{2}\right)$ in a temperature range from $343 \mathrm{~K}$ to $233 \mathrm{~K}$ showed a marked broadening of the aromatic signals together with an almost negligible downfield shift. However, in toluene- $d_{8}(9.2 \mathrm{mM})$, full coalescence of the pyridine NMR signals was observed at $253 \mathrm{~K}$ and both increasing or decreasing temperatures resulted in new environments and/or broadening (Fig. 2 and Fig. S19 to S21). While broadening at lower temperature is expected for classical supramolecular polymers, broadening or splitting at higher temperatures is an indication of an entropy-driven process $(35,36)$. Further spectroscopic evidence could be obtained from VT UV-vis and fluorescence spectroscopies (Fig. S27 and S28A). The former shows a decrease in intensity with only minor spectral changes, in agreement with other entropy-driven examples $(35,36)$, and the latter a decrease in fluorescence quantum yield at higher temperatures due to aggregation-induced fluorescence quenching. 


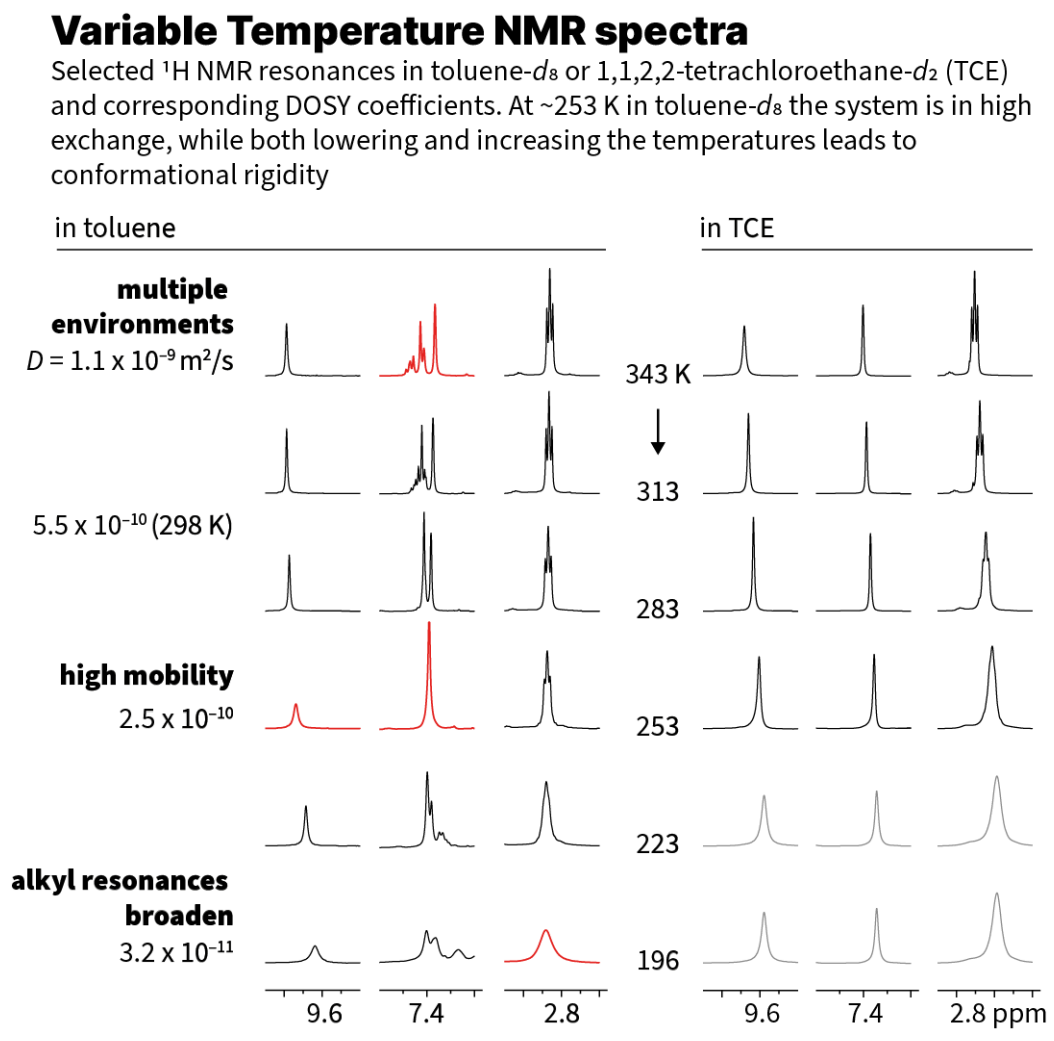

Fig. 2 Variable Temperature ${ }^{1} \mathbf{H}$ NMR of $2 \mathrm{H}-\mathrm{Car}-\mathrm{C6}$ in toluene- $\boldsymbol{d}_{\mathbf{8}}$ and TCE- $\boldsymbol{d}_{2}$. Selected regions and temperatures only, see SI for the entire datasets. In TCE broadening is only observed at low temperatures while in the aggregation-inducing solvent (toluene) distinct environments emerge at low and high temperatures. Grey signals in TCE are measured at $233 \mathrm{~K}$.

Key evidence of the carpyridine ordering and shape-assisted self-assembly was obtained by microscopy. By (scanning) transmission electron microscopy (TEM; STEM) we were delighted to observe extremely well-defined, micrometer-long 2D sheets when imaging samples prepared from 2H-Car-C6 (1 mM in toluene) (Fig. 3D). These structures were only observed for 2H-CarC6 bearing hexyl side chains and when assembled in toluene. Longer side chains (2H-Car-C12), or the absence of them (2H-Car-H), resulted in significantly less defined aggregates (Fig. S30). The results observed with 2H-Car-C12 imply that too long sidechains prevent assemblies of significant order and that interactions at the periphery of the cycles are not the dominant driving force. On the other hand, the observations for $\mathbf{2 H}-\mathbf{C a r}-\mathbf{H}$ mean that the carpyridine core is insufficient to solely drive the assembly. Higher resolution images and topologies of the 2D sheets were obtained by atomic force microscopy (AFM) as seen in Fig. 3A. Individual sheets can be seen isolated or stacked on top of one another, with highly uniform edges allowing identification of each distinct assembly (Fig. 3, B and C). In addition to single-layer sheets (Fig. S36), holes in one multi-layer sheet allowed the measurement of the thickness of a single layer, determined to be $2 \mathrm{~nm}$; a distance that is equivalent to the width of a single carpyridine molecule (Fig. 3, E and F). 
A
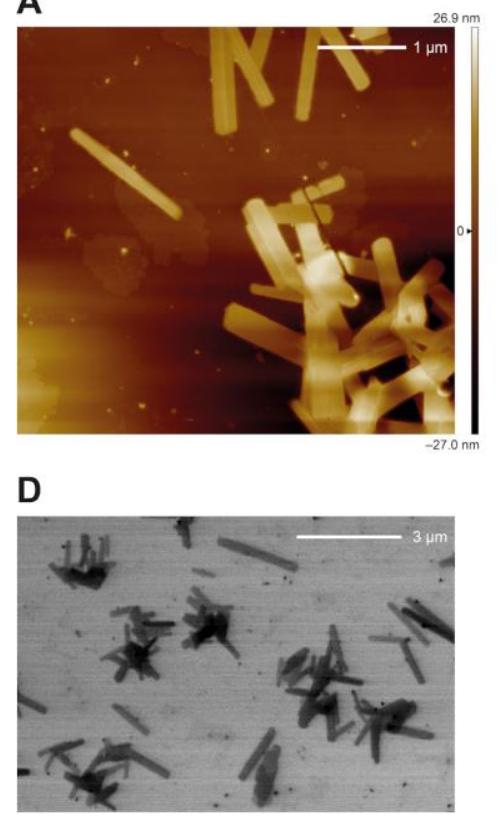

G

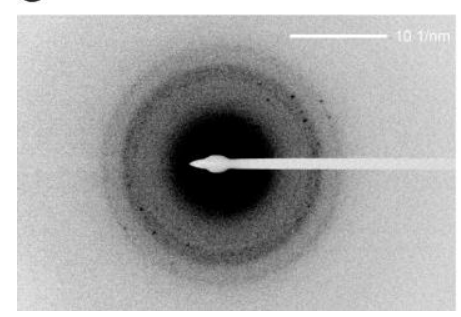

B

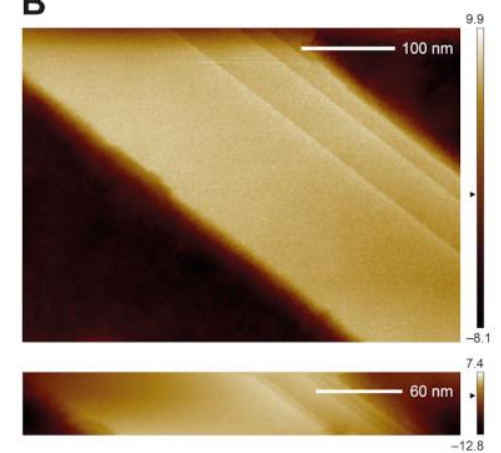

E

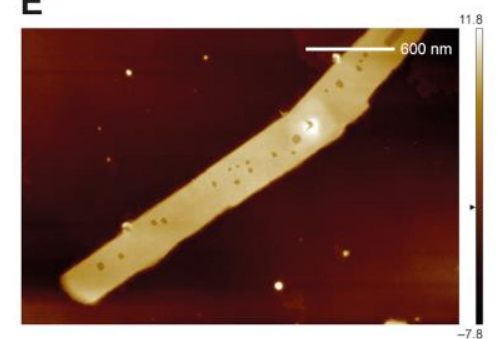

H

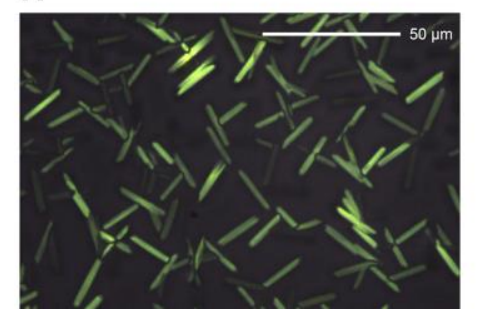

C

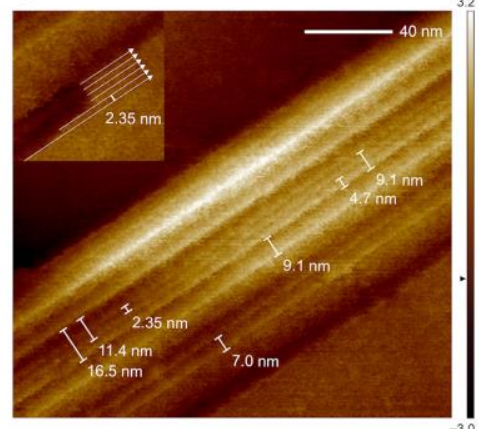

$\mathbf{F}$

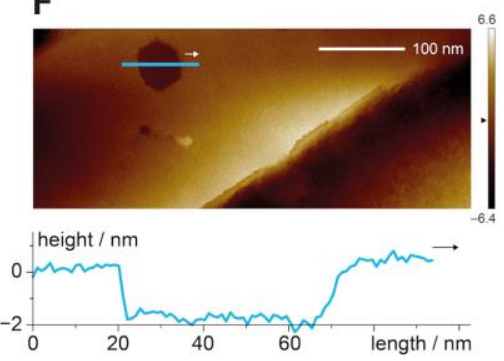

1

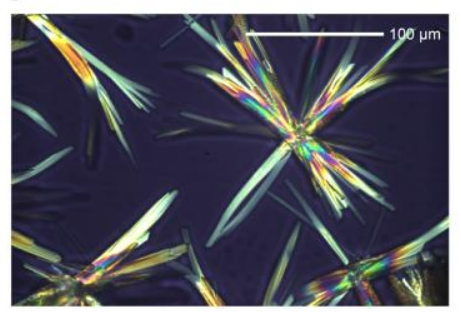

Fig. 3 Microscopic images of 2H-Car-C6 self-assembled into 2D sheets. (A) AFM images show sheets in contact with one another or isolated. (B) Sharp boundaries in multi-layer sheets allow distinction of individual units. (C) Individual columnar assemblies and their propagation direction can be distinguished in regions with defects, and by the step thickness of the edges (insert and Fig. S35). (D) STEM image showing micrometer-long individual sheets and clusters. (E and F) AFM depth measurements of a hole indicate sheet thickness of $2 \mathrm{~nm}$, consistent with that of a single molecule. (G) SAED pattern obtained from ensembles of 2D sheets. ( $\mathbf{H}$ and $\mathbf{I})$ POM images of bulk samples at different temperatures; $150{ }^{\circ} \mathrm{C}$ heating $(\mathbf{H})$ and $25^{\circ} \mathrm{C}$ after slow $\left(<5 \mathrm{~K} \cdot \mathrm{min}^{-1}\right)$ cooling from the isotropic melt $(\mathbf{I})$.

From the AFM images and the clearly defined edges, we propose that the long dimension of the assembled 2D sheets is formed by ordered stacks of carpyridines, and the side edges are mainly aliphatic contacts. Such a construction fully explains the critical interplay between both driving forces resulting in highly ordered aggregates. To confirm this hypothesis, we used the TEM sample for selected area electron diffraction (SAED) on ensembles of 2D sheets due to the rapid degradation of the organic material under the electron beam. The diffraction patterns obtained (Fig. 3G, and Fig. S34) clearly show long-range order within the structures as observed by defined diffraction spots on top of diffuse halos. We measured a principal characteristic distance of about $4 \AA$, which can be readily assigned to the $\pi-\pi$ distance between the carpyridines cores as observed by crystallography and predicted by density functional theory (DFT) calculations (Fig. 4). The observed halo pattern further emphasizes the soft-material nature of the self-assembled $2 \mathrm{D}$ sheets. 

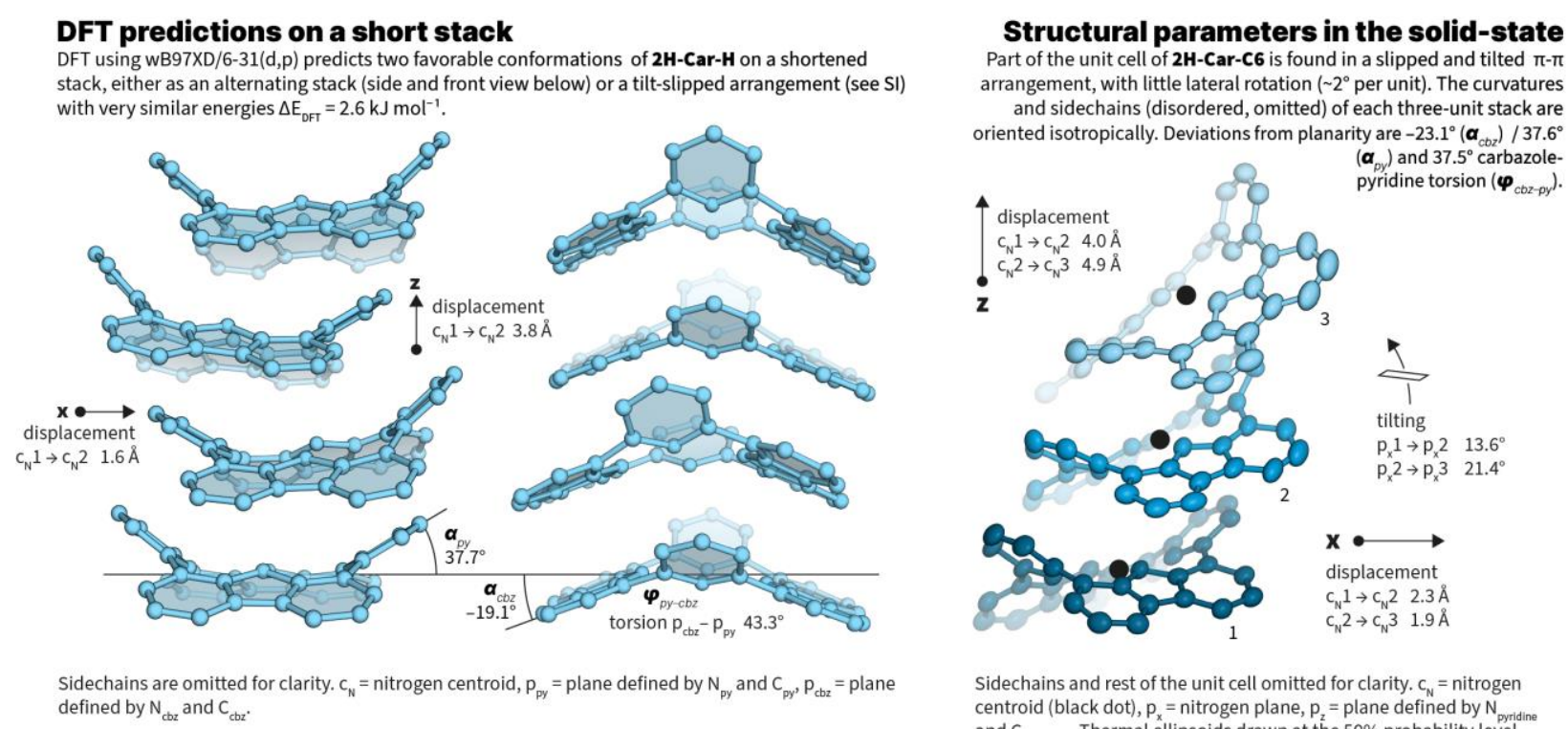

Sidechains and rest of the unit cell omitted for clarity. $c_{\mathrm{N}}=$ nitrogen centroid (black dot), $p_{x}=$ nitrogen plane, $p_{z}=$ plane defined by $N_{\text {pyridion }}$ and $\mathrm{C}_{4, \text { pyridine }}$. Thermal ellipsoids drawn at the $50 \%$ probability level.

Fig. 4 (left) DFT calculations of a four-unit stack of the carpyridine core (2H-Car-H) showing the calculated arrangement with retention of the principal axis of curvature. (right) Structural parameters derived from the X-ray crystal structure of 2H-Car-C6 showing a slipped arrangement also predicted by DFT (see SI).

Additional evidence for the proposed molecular arrangement within the 2D-sheets was obtained by single-crystal X-ray diffraction and DFT calculations. As expected, obtaining defined crystals of sufficient size in all dimensions was challenging but ultimately, single crystals of 2H-Car-C6 were obtained from a toluene/methanol mixture albeit of poor quality. Despite the heavily disordered hexyl sidechains, the obtained structures clearly show that three independent carpyridine molecules in the asymmetric unit have aligned principal axes of curvature in a 'slipped' stack. Although this arrangement is thought to be uncharacteristic of the reported 2Dsheets, it indicates that by increasing the barriers to translation and rotation of stack formation, the curvature of the saddle-shaped molecules drives the order of the assembly as postulated. Our theoretical calculations by density functional theory (DFT) using various functionals (B3LYP, wB97XD, PBE0 and the 6-31(d,p) basis set) predict that the preferred assembled configuration of the carpyridine core is an alternated stacked conformation (Fig. S42 to S44). The displaced saddle conformation as observed in the crystal structure is another local minimum but at a slightly higher energy $\left(\Delta \mathrm{E}_{\mathrm{DFT}} \sim 3 \mathrm{~kJ} \cdot \mathrm{mol}^{-1}\right)$. Although saddles are translationally displaced by $1.6 \AA$ from centroid to centroid, all carpyridines retain the same orientation along the principal axis of the stacks. In both arrangements, the predicted inter-carpyridine distance between centroids is $3.8 \AA$ and within the expected range of $\pi-\pi$ stacking effects $(37,38)$. Our DFT studies were complemented by semi-empirical calculations using the PM6 method, showing that the addition of the hexyl chains (stacks of six $\mathbf{2 H - C a r - C 6 ~ u n i t s ~ w e r e ~ s t u d i e d ) ~ l e a d s ~ t o ~ t h e ~}$ preferred stable linear alternated stacked structure proposed above (Fig. S45).

Further observations of 2H-Car-C6 in the solid-state were made with thermal analysis using differential scanning calorimetry (DSC). This showed several phase transitions and notably at least two distinct crystallization events on the cooling traces. By polarized optical microscopy, we observe at least two different (semi)crystalline phases (Fig. S39). Notably, when the cooling 
rate of the melt is carefully controlled (less than $5 \mathrm{~K} \cdot \mathrm{min}^{-1}$ ), the last crystallization transition yields large, highly uniform, sheet and needle-like crystalline regions of $>100 \mu \mathrm{m}$ in length, in strong support of a highly directional supramolecular interaction.

The simplicity of the carpyridine supramolecular units, which results in the self-assembly of large 2D sheets of single molecular thickness is unique. It is only possible because its topological elements enforce shape-assisted directional assembly - remarkably achieved in absence of directional interactions - but also enough flexibility to prevent the system from collapsing in solution like most rigid $2 \mathrm{D}$ materials. The $2 \mathrm{D}$ sheets themselves can only be assembled as a consequence of the delicate equilibrium between entropic gains and competition of both dipolar $\pi-\pi$ and hydrophobic interactions. This first and exceptional example is a direct demonstration of the power of shape in the assembly of molecular nanostructures, yet the chemical space of shapeassisting systems is largely unexplored. As the obtained assembly is a direct consequence of the underlying topography, shape-assisted self-assembly holds substantial promise as a design concept for new soft-matter materials and its implementations in nanotechnological applications such as sensing and nanofabrication.

\section{References and Notes}

1. J.-P. Behr, Ed., "The lock-and-key principle: the state of the art - 100 years on" in Perspectives in supramolecular chemistry (Wiley, New York, 1994).

2. Y. Sakurai, T. Sawada, T. Serizawa, Polym. J., in press.

3. S. Yadav, A. K. Sharma, P. Kumar, Front. Bioeng. Biotechnol. 8, 127 (2020).

4. R. P. Sijbesma, F. H. Beijer, L. Brunsveld, B. J. B. Folmer, J. H. K. K. Hirschberg, R. F. M. Lange, J. K. L. Lowe, E. W. Meijer, Science 278, 1601-1604 (1997).

5. C. Fouquey, J.-M. Lehn, A.-M. Levelut, Adv. Mater. 2, 254-257 (1990).

6. T. Aida, E. W. Meijer, S. I. Stupp, Science 335, 813-817 (2012).

7. M. Wehner, F. Würthner, Nat. Rev. Chem. 4, 38-53 (2020).

8. J. P. Hill, W. Jin, A. Kosaka, T. Fukushima, H. Ichihara, T. Shimomura, K. Ito, T. Hashizume, N. Ishii, T. Aida, Science 304, 1481-1483 (2004).

9. T. F. A. De Greef, M. M. J. Smulders, M. Wolffs, A. P. H. J. Schenning, R. P. Sijbesma, E. W. Meijer, Chem. Rev. 109, 5687-5754 (2009).

10. U. Velten, M. Rehahn, Chem. Comm. 1996, 2639-2640.

11. S. J. Rowan, J. B. Beck, Faraday Discuss. 128, 43-53 (2005).

12. W. Zhang, W. Jin, T. Fukushima, A. Saeki, S. Seki, T. Aida, Science 334, 340-343 (2011).

13. T. Fukino, H. Joo, Y. Hisada, M. Obana, H. Yamagishi, T. Hikima, M. Takata, N. Fujita, T. Aida, Science 344, 499-504 (2014).

14. F. Herbst, S. Seiffert, W. H. Binder, Polym. Chem. 3, 3084-3092 (2012).

15. N. J. Van Zee, B. Adelizzi, M. F. J. Mabesoone, X. Meng, A. Aloi, R. H. Zha, M. Lutz, I. A. W. Filot, A. R. A. Palmans, E. W. Meijer, Nature 558, 100-103 (2018).

16. U. Lewandowska, W. Zajaczkowski, S. Corra, J. Tanabe, R. Borrmann, E. M. Benetti, S. Stappert, K. Watanabe, N. A. K. Ochs, R. Schaeublin, C. Li, E. Yashima, W. Pisula, K. Müllen, H. Wennemers, Nat. Chem. 9, 1068-1072 (2017).

17. Y. Li, X. Dong, M. Yu, W. Liu, Y. Nie, J. Zhang, L. Xie, C. Xu, J. Liu, W. Huang, Small 17, 2102060 (2021).

18. Y. Lin, M. R. Thomas, A. Gelmi, V. Leonardo, E. T. Pashuck, S. A. Maynard, Y. Wang, M. M. Stevens, J. Am. Chem. Soc. 139, 13592-13595 (2017).

19. Y. Wang, X. Feng, M. Lee, Org. Chem. Front. 8, 3681-3685 (2021). 
20. N. Zhang, T. Wang, X. Wu, C. Jiang, T. Zhang, B. Jin, H. Ji, W. Bai, R. Bai, ACS Nano. 11, 7223-7229 (2017).

21. M. B. Avinash, T. Govindaraju, Adv. Funct. Mater. 21, 3875-3882 (2011).

22. J. Kang, D. Miyajima, T. Mori, Y. Inoue, Y. Itoh, T. Aida, Science 347, 646-651 (2015).

23. M. Ueda, T. Aoki, T. Akiyama, T. Nakamuro, K. Yamashita, H. Yanagisawa, O. Nureki, M. Kikkawa, E. Nakamura, T. Aida, Y. Itoh, J. Am. Chem. Soc. 143, 5121-5126 (2021).

24. K. Kato, K. Takaba, S. Maki-Yonekura, N. Mitoma, Y. Nakanishi, T. Nishihara, T. Hatakeyama, T. Kawada, Y. Hijikata, J. Pirillo, L. T. Scott, K. Yonekura, Y. Segawa, K. Itami, J. Am. Chem. Soc. 143, 5465-5469 (2021).

25. L. Verstraete, S. De Feyter, Chem. Soc. Rev. 50, 5884-5897 (2021).

26. J. Chen, E. Zhu, J. Liu, S. Zhang, Z. Lin, X. Duan, H. Heinz, Y. Huang, J. J. De Yoreo, Science. 362, 1135-1139 (2018).

27. S. Xiao, J. Tang, T. Beetz, X. Guo, N. Tremblay, T. Siegrist, Y. Zhu, M. Steigerwald, C. Nuckolls, J. Am. Chem. Soc. 128, 10700-10701 (2006).

28. X. Feng, V. Marcon, W. Pisula, M. R. Hansen, J. Kirkpatrick, F. Grozema, D. Andrienko, K. Kremer, K. Müllen, Nat. Mater. 8, 421-426 (2009).

29. A. S. Filatov, L. T. Scott, M. A. Petrukhina, Cryst. Growth Des. 10, 4607-4621 (2010).

30. R. Renner, M. Stolte, F. Würthner, ChemistryOpen. 9, 32-39 (2020).

31. Y. Sakamoto, T. Suzuki, J. Am. Chem. Soc. 135, 14074-14077 (2013).

32. J. I. Urgel, M. Di Giovannantonio, Y. Segawa, P. Ruffieux, L. T. Scott, C. A. Pignedoli, K. Itami, R. Fasel, J. Am. Chem. Soc. 141, 13158-13164 (2019).

33. F. J. Baur, H. K. Hawley, US3498798A (1970).

34. L. Arnold, H. Norouzi-Arasi, M. Wagner, V. Enkelmann, K. Müllen, Chem. Commun. 47, 970-972 (2011).

35. D. Görl, F. Würthner, Angew. Chem. Int. Ed. 128, 12273-12277 (2016).

36. P. Dey, P. Rajdev, P. Pramanik, S. Ghosh, Macromolecules 51, 5182-5190 (2018).

37. Y. Guan, M. L. Jones, A. E. Miller, S. E. Wheeler, Phys. Chem. Chem. Phys. 19, 1818618193 (2017).

38. L.-J. Riwar, N. Trapp, B. Kuhn, F. Diederich, Angew. Chem. Int. Ed. 56, 11252-11257 (2017).

Acknowledgments: Imaging was performed with support of the Center for Microscopy and Image Analysis, University of Zurich, and the microscopy facility at the ICS, Strasbourg. We also thank S. Jurt, T. Fox and N. Bross for assistance with NMR measurements, the Mass Spectrometry Laboratory at the University of Zurich for MS measurements, K. Feldman for assistance with DSC measurements, G. Patzke for DLS measurements, P. Zwick and A. Wildi for helpful discussions and K. Gademann for generously hosting and supporting our research group.

Funding: $\quad$ Swiss National Science Foundation grant PZ00P2_180101 (MR)

University of Zurich Forschungskredit (JFW)

\section{Author contributions:}

Conceptualization - JFW, LG, AVJ, MR 
Formal Analysis - JFW, LG, AVJ, MR

Funding acquisition - JFW, MR

Investigation - JFW, LG, PP, MM, AVJ, MR

Methodology - JFW, LG, AVJ, MM, MR

Project administration - MR

Resources - AVJ, MR

Supervision - MR

Validation - JFW, LG, AVJ, MR

Visualization - JFW, LG, AVJ, MR

Writing - original draft - JFW, LG, AVJ, MR

Writing - review \& editing - JFW, LG, AVJ, MR

Competing interests: The authors declare that they have no competing interests.

Data and materials availability: All data are available either in the main text, the supplementary materials or by request of the corresponding author. 